\title{
DOI:10.22374/jeleu.v3i3.99 \\ ASPECTS OF UROLOGICAL MANAGEMENT AFFECTED BY THE CORONAVIRUS PANDEMIC
}

Editorial

\author{
Daron Smith ${ }^{1}$ MA, BMBCh, MD, FRCS(Urol) and Wasim Mahmalji ${ }^{2}$ MBBS, BSc, MSc, MRCS, FRCS \\ ${ }^{1}$ Consultant Endoluminal EndoUrologist, University College Hospital, London, UK \\ ${ }^{2}$ Consultant Urological Surgeon, Wye Valley NHS Trust, England, UK
}

Corresponding Authors: daron.smith1@nhs.net. wasim.mahmalji@gmail.com

Submitted: August 21, 2020. Published: August 23, 2020.

\begin{abstract}
As 2020 enters its final third, it is long since evident that the COVID-19 pandemic has had an extraordinary impact throughout the world, presenting new and complex challenges. This is the case both for the treatment of the SARS-CoV-2 severe respiratory illness itself and also for the "knock-on" effects this has had for the contemporary management of existing or incident non-respiratory illnesses.
\end{abstract}

In this special COVID-19 edition of JELEU, we start with three articles that discuss aspects of urological management that have been affected by the coronavirus pandemic: the impact of living with cystinuria, the challenges of delivering a urodynamic service, and the consequences of adding a CT chest to a CTKUB for patients with pain suggestive of ureteric colic.

Using patient-reported data from Guy's Hospital's specialist cystinuria clinic, Chalokia et al have shown that the ability of the patient to access medications and/or routine clinic appointments has been adversely affected, including over half of the patients who had acute colic episodes but who were afraid to seek medical attention despite their pain. Interestingly, just under a quarter of the patients found their fluid intake to be easier to manage during a lockdown, a salutary thought to keep in mind amongst the pressures of returning to a "new normal" working week as the recovery phase of lockdown easing continues.

Abdelmotagly and colleagues report their findings of an additional CT chest for any patient who presented to the emergency department during the pandemic with pain requiring abdominal CT. Although $9 \%$ of patients had CT changes suggestive of COVID, their subsequent management was not affected, nor was the outcome of their disease, at the "cost" of the additional radiation exposure of the wider imaging field. This study highlights the challenge of rapidly emerging advice during a time of imperfect knowledge of the natural history of the COVID-19 disease and without the availability of a "gold standard" rule-in / rule-out diagnostic test for its presence.

In the third article in this series, Jabbar et al report their survey of an international cohort of clinicians regarding their urodynamics service during the pandemic, with particular attention on the standard practice of cough-tests during the study. The implications for the high-quality delivery of UDS, such as pre-procedure isolation and COVID testing to allow the patient to cough, and thereby maintaining the calibration and accuracy of the test, whilst leaving adequate time between patients for cleaning necessarily affects the throughput of the service. Furthermore, the implications of performing elective diagnostic tests for which subsequent surgical procedures may be "low-level" on the priority list during times of scarce theatre time need to be considered.

The unifying theme of all three articles is the anxiety and uncertainty for patients and physicians alike during the pandemic and the benefits of clear and unambiguous advice for the appropriate and safe delivery of medical care during and after it. This applies to patient-facing information regarding the need for selfisolation/shielding and testing before procedures, as well as the more complex interplay between the patient's urological condition, the prevalent likelihood of contracting COVID-19 at the time of their scheduled hospital attendance, and their individual risk/severity of morbidity from actually contracting it. 
The article on UDS provides a useful triage form that can be adapted to the needs of individual units, or even adapted for different diagnostic tests/conditions: the key is that all future medical decisions are likely to require more risk/benefit stratification than has been needed in the past. This takes time, and requires holistic knowledge of the patient, the local hospital infrastructure, and must be supported by the ongoing research into COVID-19 diagnostics, treatment, and prevention.

Healthcare systems across the world have had their financial and human resources stretched to deliver rapid adaptations to clinical work that are still needed. We hope that the articles in this special edition will be helpful to stimulate further thought for the safe reintroduction of urological services as the period of post-COVID-19 recovery continues. 